\section{L'Actualité économique}

L'ACTUALITÉ

ÉCONOMIQUE

\section{Inventaire des documents relatifs à l'histoire économique du Canada français - 4}

\section{Cameron Nish}

Volume 43, numéro 4, janvier-mars 1968

URI : https://id.erudit.org/iderudit/1003121ar

DOI : https://doi.org/10.7202/1003121ar

Aller au sommaire du numéro

Éditeur(s)

HEC Montréa

ISSN

0001-771X (imprimé)

1710-3991 (numérique)

Découvrir la revue

Citer ce document

Nish, C. (1968). Inventaire des documents relatifs à l'histoire économique du Canada français - 4. L'Actualité économique, 43(4), 789-808.

https://doi.org/10.7202/1003121ar
Ce document est protégé par la loi sur le droit d'auteur. L'utilisation des services d'Érudit (y compris la reproduction) est assujettie à sa politique d'utilisation que vous pouvez consulter en ligne.

https://apropos.erudit.org/fr/usagers/politique-dutilisation/ 


\title{
Inventaire des documents relatifs à l'histoire économique du Canada français - $4^{\star}$
}

\author{
1748 \\ C $11 \mathrm{~A}$
}

118. Ouyatanons, 10 janvier 1748. «Memoire des fournitures fait par moy Claude Dudevoir au poste des ouyatanons Pour Le Compte Et Service du Roy Suivant Les ordres de Mr le Chevalier de Laperade Commandant pour le Roy au dit Lieux. » (Diverses fournitures.) Signé Claude Dudevoir, Lapérade fils, La Galissio nière, Bigot. f 249-250, pp. 324327.

118. Miamis, 16 janvier 1748. «Certificat. 》 (Fournitures.) Signé du Buisson, La Galissionière, Bigot. f 295, p. 29.

118. Miamis, 29 janvier 1748. «Certificat. » (Gages.) Signé du Buisson, La Galissionière, Bigot. f 296, p. 30.

118. St. Joseph, 30 janvier 1748. «Certificat. » (Gages). Signé Piqueté, La Galissionière, Bigot. f 269, p. 356.

118. Ouyatanons, 6 février 1748. «Certificat.» (Gages.) Signé Lapérade fils, La Galissionière, Bigot. f 290, p. 24.

118. Ouyatanons, 8 février 1748. «Certificat.» (Diverses fournitures.) Signé Lapérade, La Galissionière, Bigot. f 190, p. 250.

118. S. Joseph, 12 février 1748. «Certificat.» (Gages.) Signé Piqueté, La Galissionière, Bigot. f 263, p. 350 .

- Pour les trois premières parties de ce document, voir L'Actualité Économique, avril-juin, juillet-septembre et octobre-décembre 1967 . 


\section{LACTUALITE ECONOMIQUE}

118. St. Joseph, 12 février, 1748. «Certificat.» (Gages.) Signé Piqueté, La Galissionnière, Bigot. f 262, p. 349.

118. Montréal, 16 février 1748. «Certificat. » (Fournitures.) Signé La Verendrye, La Galissionière, Bigot. f 230, p. 302.

116-1. Versailles, 25 février 1748. «Pour les Depenses de Canada pend't L'année 1748. » (Construction et radoub de bateaux et ca' nots ; fret et voiture ; journées d'ouvriers ; achats de marchandises ; présents aux Sauvages; bois à brûler ; subsistance et remèdes ; dé, penses imprévues ; appointements des officiers généraux et autres ; appointements des officiers reformés; solde des compagnies; gages et. entretien des employés: Québec, Trois-Rivières, Montréal ; loyers de magasins et bureaux : Québec, Montréal; gratifications ordinaires, extraordinaires : Québec, Trois-Rivières, Montréal ; dépenses pour préparatifs de guerre; fortifications; autres diverses dépenses ; appointements et solde des compagnies de l'Isle Royalle ; récapitulation.) f 56-64, pp. 92-108.

118. Ouyatanons, 30 février 1748. «Estat des fournitures faites au Roy pour les 8yatanons Livrées au nommé Godore et proux suivant L'ordre qu'ils nous ont representé du D La Chibe Chargé aud Poste. » (Diverses fournitures.) Signé Lapérade, La Galissionière, Bigot. f 233, p. 305.

118. Détroit, 13 mars 1748. «Certificat.» (Fournitures.) Signé Piquoté, La Galissionière, Bigot. f 234, p. 306.

118. Ouyatanons, 15 mars 1748. «Certificat.» (Gages.) Signé Lapérade fils, La Galissionière, Bigot. f 280, p. 13.

118. Ouyatanons, 20 mars 1748. «Certificat.» (Gages.) Signé Lapérade fils, La Galissionière, Bigot. f 279, p. 12.

118. St. Joseph, 23 mars 1748. «Certificat. 》 (Fournitures.) Signé Piquoté de Belestre, La Galissionière, Bigot. f 189, p. 249.

118. St. Joseph, 23 mars 1748. «Certificat. 》(Gages.) Signé Piquoté, La Galissionière, Bigot. f 268, p. 355.

118. Ouyatanons, 4 avril 1748. « Certificat. 》 (Fournitures.) Signé Lapérade fils, La Galissionière, Bigot. f 215, p. 285. 
118. Ouyatanons, 4 avril 1748. «Certificat. 》(Gages.) Signé La pérade fils, La Galissionière, Bigot. f 282, p. 15.

118. St. Joseph, 12 avril 1748. «Memoire des fournitures faittes par nous Marin Laperriere Et de quindre Chargés des ordres de Monsieur le Général a 16 hommes tant françois que sauvage pour aller Chercher Monsieur Delestre au détroit. 》(Diverses fournitures.) Signé Dagneaux de Quindre, Laperrière Marin, Piquoté, La Galissionière, Bigot. f 235, pp. 307-308.

118. St. Joseph, 14 avril 1748. «Memoire des fournitures faite au Roy par Nous Marin La Perriere et de Quindre pa rordre de $\mathrm{Mr}$ de Belestre. 》 (Diverses fournitures.) Signé Dagneaux de Quindre, Laperrière Marin, Piquoté, La Galissionière, Bigot. f 284, pp. 17-18.

118. St. Joseph, 16 avril 1748. «Memoire des fournitures faitte au Roy par Moy Porlier La Groizardiere par ordre de Monsieur le Commandant de St Joseph Mess'rs Marin \& Dequindre n'en ayant point. » (Diverses fournitures.) Signé Porlier de Groizardière, $\mathrm{Pi}$ quoté de Belestre, La Galissionière, Bigot. f 212-214, pp. 279-284.

118. St. Joseph, 16 avril 1748. «Certificat. » (Fournitures.) Signé Laperrière Marin, Piquoté de Belestre, La Galissionière, Bigot. f 229 , p. 301.

118. St. Joseph, 17 avril 1748. «Certificat. 》 (Fournitures.) Signé Piquoté de Belestre, La Galissionière, Bigot. f 192, p. 252.

118. Miamis, 23 avril 1748. «Certificat. » (Gages.) Signé du Buisson, La Galissionière, Bigot. f 292, p. 26.

118. Ouyatanons, 24 avril 1748. « Estat des fournitures faites par moy Louis clairmon par L'ordre de $\mathrm{mr}$ Lechevalier de la perade Commendant audit Lieux le 31 janvier 1748. 》 (Diverses fournitures.) Signé Lapérade, La Galissionière, Bigot. f 257, pp. 344-345.

118. St. Joseph, 25 avril 1748. «Certificat. » (Fournitures.) Signé Piquoté, La Galissionière, Bigot. f 232, p. 304.

118. Ouyatanons, 25 avril 1748. «Certificat.» (Gages.) Signé Piquoté, La Galissionière, Bigot. f 242, p. 316. 
118. Ouyatanons, 27 avril 1748. «Certificat.» (Fournitures.) Signé Lapérade fils, La Galissionière, Bigot. f 289, p. 23.

118. Ouyatanons, 3 mai 1748. «Estat des ouvrages et fournitures faittes pour les piauquichias ou vermillon pour le Compte du Roy par l'ordre de monsieur le chevallier de Laperade Commandant pour le Roy au poste des ouyatanons fait par moy françois Bauchasse forgeron du vermillon. 》 (Diverses fournitures et ouvrages.) Signé S. Beauchaine, Lapérade, La Galissionière, Bigot. f 204, p. 266.

118. Ouyatanons, 6 mai 1748. «Certificat. » (Gages.) Signé Lapérade fils, La Galissionière, Bigot. f 281, p. 14.

118. Ouyatanons, 8 mai 1748. «Certificat. 》 (Fournitures.) Signé Lapérade fils, La Galissionière, Bigot. f 286, p. 20.

118. Ouyatanons, 16 mai 1748. «Memoire des fournitures que moy Leclair a faittes En Vermillion pour le Compte Et service du Roy par l'ordre de monsieur Lechevaillier de Laperade Commandant au poste des ouyatanons Commencé le 13 avril 1748. »(Diverses fournitures.) Signé François Leclair, Lapérade fils, La Galis' sionière, Bigot. f 186, pp. 245-256.

118. Ouyatanons, 18 mai 1748. "Mémoire des fournitures faites par moy Michel Gamelin au poste des 8ytanons pour le Compte et Service du Roy par l'ordre de M le chevalier de laperade Commandant pour le Roy aud Poste Et Suivant le Recommandations de $\mathrm{M}$ le Chev. de Berthel Commandant au poste des yllinois qu'il estoit necessaire de menager les Nations d'8abache. 》 (Diverses fournitures.) Signé Michel Gamelin, Lapérade fils, La Galissionière, Bigot. f 273-274, pp. 3 à 7.

118. 20 mai 1748. «Fourniture faite par moy charles Courtois aux quatres nassuions sauvages. 》(Diverses fournitures à des officiers et à des sauvages.) f 305-326, pp. 41-87.

118. Miamis, 29 mai 1748. «Certificat.» (Fournitures.) Signé Lapérade fils, La Galissionière, Bigot. f 191, p. 251.

118. Michilimackinac, 2 juin 1748. "Memoire de ce que moy francois Menard Interprette pour le Roy a missilimakinac ay fourni 
au Crapeau Pouti8atamis, par l'ordre de monsieur de St. Pierre qui Lavoit fait relacher En chemin pour Montreal L'automme der' niere Etant a son Village pour L'Execution des ordres de monsieur le general. » (Diverses fournitures.) Signé Legardeur de St. Pierre, La Galissionière, Bigot. f 216, p. 286.

118. St. Joseph, 4 juin 1748. «Memoire des fournitures faites au Roy par moy Cejard Dagneau de Quindre par ordre de M le Commandant a St. Joseph. » (Diverses fournitures.) Signé Piquoté de Belestre, La Galissionière, Bigot. f 194, p. 254.

118. St. Joseph, 5 juin 1748. «Memoire des fournitures faite au Roy par moy Porlier La Groizardiere par ordre de m. Le Commandant. » (Diverses fournitures.) Signé Piquoté de Belestre, La Galissionière, Bigot. f 225, pp. 297-298.

118. St. Joseph, 5 juin 1748. «Certificat.» (Fournitures.) Signé Piquoté, La Galissionière, Bigot. f 231, p. 303.

118. Détroit, 8 juin 1748. «Certificat. » (Gages.) Signé du Buis son, La Galissionière, Bigot. f 294, p. 28.

118. Miamis, 10 juin 1748. «Memoire des Marchandises que moy Jean Baptiste Godefroy Vieuxpont ait fourny pour le compte et Service du Roy par ordre de Monsieur Dubuisson officier et Commandant pour le Roy au miamis. » (Diverses fournitures.) Signé Godefroy, du Buisson, Bigot. f 97-102, pp. 117-127.

118. A la Baye, 11 juin 1748. «Mémoire des fournitures faites pour le service du Roy par Les fermiers de la Baye par ordre de Monsieur de Verchere Commandant dud't Poste pour les presents faits aux Scioux. » (Diverses fournitures; pourcentage du bénéfice.) Signé Clignancourt, Verchères, La Galissionière, Bigot. $f$ 199-200, pp. 260-262.

118. A la Baye, 11 juin 1748. «Memoire des fournitures faites pour le service du Roy par Les fermiers de la Baye par ordre de Mons'r de Vercheres Commandant dud. Poste. » (Diverses fournitures; pourcentage de bénéfice.) Signé Clignancourt et Compagnies, Verchères, La Galissionière, Bigot. f 201, pp. 263-264.

118. A la Baye, 11 juin 1748. «Memoire des fournitures faites 
pour le service du Roy par les fermiers de la Baye par ordre de Monsieur de Vercheres Commandant dud Poste pour les presents faits aux Scioux. 》 (Diverses fournitures; pourcentage de bénéfice.) Signé Clignancourt et Compagnies, Verchères, La Galissio nière, Bigot. f 299-300, pp. 33-35.

118. A la Baye, 12 juin 1748. «Memoire des fournitures faites pour le service du Roy par Les fermiers de la Baye par ordre de Monsieur de Vercheres Commandant au Poste. » (Diverses four' nitures.) Signé Clignancourt et Compagnies, Verchères, La Galissionière, Bigot. f 264-265, pp. 351-353.

118. St. Joseph, 13 juin 1748. «Certificat. 》 (Fournitures.) Signé Piquoté de Belestre, La Galissionière, Bigot. f 193, p. 253.

119. Michilimackinac, 14 juin 1748. «Certificat. 》 (Gages.) Signé Verchères, La Jonquière, Bigot. f 176, p. 166.

118. Détroit, 12 juin 1748. «Memoire des ouvrages de Coutures fait par moy femme de Barrois pour le service du Roy suivant les ordres de monsieur de Longueuil. » (Divers travaux.) Signé Na. varre, Longueuil, La Jonquière, Bigot. f 378, pp. 146-148.

119. A la Baye, 18 juin 1748. «Memoire de l'ouvrages de la forge des Sakis fait par nous fermiers de la Baye aux Sakis Renards et Puants. » (Divers travaux.) Signé Jean Garreau et Compagnies, Verchères, La Jonquière, Bigot. f 21, p. 28.

118. Détroit, 18 juin 1748. « Certificat pour Gauché.» (Diverses fournitures.) Signé Longueuil, Navarre, La Galissionière, Bigot. f 270 , p. 357 .

119. A la Baye, 18 juin 1748. «Memoire de L'Ouvrage de la forge de la folle-avoines, faite par nous fermiers de La Baye aux Folles avoines et Puants. » (Divers travaux.) Signé Garreau et Compagnies, Verchères, La Jonquière, Bigot. f 22, p. 29.

118. Michilimackinac, 23 juin 1748. «Certificat. » (Diverses fournitures.) Signé Legardeur de St. Pierre, La Galissionière. f 303, p. 38.

118. Michilimackinac, 25 juin 1748. « Certificat. » (Fournitures.) Signé Verchères, La Galissionière, Hocquart. f 239, p. 312. 
118. Michilimackinac, 25 juin 1748. «Certificat. 》 (Fournitures.) Signé Verchères, La Galissionière. f 302, p. 37.

118. Michilimackinac, 26 juin 1748. «Certificat. 》 (Fournitures.) Signé Verchères, La Galissionière, Bigot. f 203, p. 265.

118. Michilimackinac, 26 juin 1748. «Certificat. 》(Fournitures.) Signé Verchères, La Galissionière, Bigot. f 223, p. 296.

118. Michilimackinac, 26 juin 1748. « Certificat. 》 (Fournitures.) Signé Le Gardeur de St. Pierre, Hocquart. f 236, p. 309.

118. Michilimackinac, 26 juin 1748. «Certificat. » (Gages.) Signé Verchères, La Galissionière, Hocquart. f 237, p. 310.

118. Détroit, 29 juin 1748. «Fournitures par Metivier. » (Diverses fournitures.) Signé Metivier, Longueuil, Navarre, La Galis' sionière, Bigot. f 228, p. 300 .

118. Michilimackinac, 26 juin 1748. «Certificat. » (Gages.) Signé Le Gardeur de St. Pierre, Hocquart. f 238, p. 311.

118. Détroit, 29 juin 1748. «Fournitures par Jean Claude Ni, neau. » (Diverses fournitures.) Signé Longueuil, Navarre, La Jonquière, Bigot. f 366, p. 131.

118. Détroit, 30 juin 1748. «Fournitures, faites par Jorian \& Com pagnies au detroit, pour le Compte et service du Roi, suivant les ordres et demandes de Monsieur le Chevallier Delongueuil commandant pour le Roi aud. lieu, comme ciaprès. » (Diverses fournitures.) Signé Bondy, Douville, La Galissionière. f 125-127, pp. 163-168.

118. Détroit, 1 juillet 1748. «Certificat.» (Fournitures.) Signé du Buisson, La Galissionière, Bigot. f 283, p. 16.

118. Détroit, 3 juillet 1748. «Memoire des fournitures faite par moy Carrignan au Detroit pour le service du Roy dans les fifférentes occasions Expliquées cy‘après. » (Diverses fournitures.) Signé $\mathrm{Na}$ varre, Longueuil, La Jonquière, Bigot. f 372-373, pp. 138-140.

118. (Détroit), 7 juillet 1748. «Fournitures par pierre forville. » (Diverses fournitures.) Signé Forville, Navarre, Longueuil, Bigot. f 218 , p. 288. 
118. Détroit, 8 juillet 1748. «Certificat. 》 (Diverses fournitures.) Signé Pierre Saint Cosme, Longueuil, Navarre, La Galissionière, Bigot. f 136, p. 177.

118. Détroit, 9 juillet 1748. «Memoire des ouvrages de Menuiserie faittes par Moy Nicolas Lasette au detroit sur La demande de Monsieur de Longueuil pour le Compte et Service du Roy. » (Divers travaux.) Signé Longueuil, Navarre; La Galissionière, Bigot. f 246-247, pp: 320-322.

118. Détroit, 9 juillet 1748. «Ouvrages faits par Moy hiacinthe Reaume au detroit pour Le Service du Roy Suivant Les ordres de Monsieur le Chevalier de Longueuil. Commandant. » (Divers travaux.) Signé Navarre, Longueuil, La Galissionière, Bigot. f 288, p. 22.

118. Ouyatanons, 10 juillet 1748. «Certificat. 》 (Fournitures.) Signé Lapérade, La Galissionière, Hocquart. f 240, p. 313.

118. (Détroit), 10 juillet 1748. « Fournitures par Pierre Vallée. » (Diverses fournitures.) Signé Navarre, Longueuil, La Galissionière, Bigot. f 2:43, p. 317.

118. Détroit, 10 juillet 1748. «Memoire des ouvrages que j’ay fait par ordre de monsieur le Commandant pour le Roy du Depuis Le mois d'octobre de l'année 1747 pour les Sauteux, Les autroix et les Epous. » (Divers travaux.) Signé Desbuttes, Longueuil, Navarre, La Galissionière, Bigot. f 252-256, pp. 329-343.

118. Détroit, 10 juillet 1748. «Memoire des ouvrages de Maçonnerie que j'ai faits pour le Service du Roy au Détroit Moy francois Janisse Par ordre de $M$ de Longueuil. » (Divers travaux.) Signé Longueuil, Navarre, La Jonquière, Bigot. f 364, p. 129.

118. Détroit, 11 juillet 1748. «Certificat. » (Diverses fournitures.) Signé Navarre, Longueuil, La Galissionière, Bigot. f 195, p. 255.

118. Détroit, 12 juillet 1748. « Certificat pour Bondy. » (Diverses fournitures.) Signé Bondy, Navarre, La Galissionière, Bigot. f 49, pp. 66-67.

118. Détroit, 13 juillet 1748. «Certificat.» (Gages.) Signé Longueuil, La Galissionière, Bigot. f 245, p. 319. 
118. Détroit, 13 juillet 1748. «Memoire des fournitures faites par moy Chevallier au Roy par ordre de Monsieur de Longueuil. » (Diverses fournitures.) Signé Chevalier, Navarre, Longueuil, La Galissionière, Bigot. f 197, p. 258.

118. Détroit, 14 juillet 1748. «Certificat. 》 (Diverses fournitures.) Signé Pierre St. Cosme, Longueuil, Navarre, La Galissionière, Bigot. f 135 , p. 176.

118. Détroit, 14 juillet 1748. «Certificat pour Claude Lesprit. 》 (Diverses fournitures.) Signé Navarre, Longueuil, La Galissionière. Bigot. f 196, pp. 256-257.

118. Détroit, 14 juillet 1748. «Certificat pour Claude Gouin.» (Diverses fournitures.) Signé Claude Gouin, Longueuil, Navarre, La Galissionière, Bigot. f 260, p. 348.

118. Détroit, 14 juillet 1748. «Certificat. 》 (Fournitures.) Signé Longueuil, Navarre, La Galissionière, Bigot. f 285, p. 19.

118. Détroit, 15 juillet 1748. «Certificat. 》 (Diverses fournitures.) Signé Deruisseaux, Navarre, Longueil, La Galissionière, Bigot. f 187 , p. 247.

118. Détroit, 15 juillet 1748. «Certificat pour Louis Campeau. » (Diverses fournitures.) Signé Louis Campeau, Navarre, Longueuil, La Galissionière, Bigot. f 287, p. 21.

118. Détroit, 16 juillet 1748. «Ouvrages faits par moy Janisse au detroit p'r Le Service du Roy par et suivant Les ordres de Mr Le Chevalier de Longueuil Commandant audit Lieu Comme Cy après. » (Divers travaux.) Signé Janisse, Longueuil, Navarre, La Galissionière, Bigot. f 198, p. 259.

118. Détroit, 16 juillet 1748. «Memoire des ouvrages darmueir que moy andré Barthe ay fait par L'ordre de $\mathrm{M}$ De Longueuil Commandant au detroit Suivant Les Billets a luy Remis a Commances depuis Le deux may 1748 Jusqu'au douze juillet de la meme année. » (Divers travaux et fournitures.) Signé Barthe, Longueil, Navarre, La Galissionière, Bigot. f 219-222, pp. 289-295.

118. Détroit, 16 juillet 1748. «Memoire des ouvrages darmurie et de forge que Moy andré Barthe a fait pour Le Service du Roy 
par ordre de Monsieur de Longueuil Commandant au detroit a Commancer depuis Le 24 oct'bre jusqu'au 29 avril 1748. » (Divers travaux et fournitures.) Signé Barthe, Longueuil, Navarre, $\mathrm{La} \mathrm{Ga}$ lissionière, Bigot. f 271-272, pp. 358-359 et 1-2.

118. Détroit, 17 juillet 1748. «Certificat.» (Fournitures.) du Buisson, Bigot. f 227, p. 299.

118. Détroit, 17 juillet 1748. «Memoire des Charriages de Bois de Chauffage et Autres faits par moi Pierre Labadie pour le Service du Roy suivant les ordres de M. de Longueuil au Detroit. » (Divers travaux.) Signé Labadie, Longueuil, Navarre, La Galissionière, Bigot. f 241, pp. 314315 .

118. Détroit, 17 juillet 1748. «Certificat pour Jacques Campeau. 》 (Diverses fournitures.) Signé Jacques Campeau, Navarre, Longueuil, La Galissionière, Bigot. f 291, p. 25.

118. Détroit, 17 juillet 1748. « Certificat pour Jacques Cardinal. » (Diverses fournitures.) Signé Navarre, Longueuil, La Galissionière, Bigot. f 297, p. 31.

118. Détroit, 17 juillet 1748 . «Memoire des fusils que J'ay racommodé par ordre de Monsieur de Celoron Commandant de L'Excorte. » (Divers travaux.) Signé St. Martin Dubutte, Céloron, La Galissionière, Bigot: f 304 , pp. 39-40.

118. Détroit, 17 juillet 1748. «Certificat. 》 (Fournitures.) Signé Navarre, Longueuil, La Galissionière, Bigot. f 395, p. 207.

118. Détroit, 17 juillet 1748. «Certificat. » (Diverses fournitures.) Signé Navarre, Longueuil, La Jonquière, Bigot. f 396, p. 208.

118. Détroit, 18 juillet 1748. «Certificat.» (Fournitures.) Célo ron, La Galissionière, Bigot. f 188, p. 248.

118. Détroit, 18 juillet 1748. «Certificat pour St. André. 》 (Diverses fournitures.) Signé Navarre, Longueuil, La Galissionière, Bigot. f 251, p. 328.

118. Ouyatanons, 24 juillet 1748. «Memoire des ouvrages que j'ay faittes au sauvages pour le Compte et service du Roy par lordre de monsieur de la perade Commendant pour le Roy aux 
ouyatanons Commencer 12 eie Juin 1748. » (Divers travaux et fournitures.) Signé Charles Bonneaux, Lapérade, La Galissionière, Bigot. f 217, p. 287.

119. Ouyatanons, 24 juillet 1748. «Certificat. 》 (Gages.) Signé Lapérade, La Jonquière, Bigot. f 64, p. 65 .

119. St. Joseph, 26 juillet 1748. «Memoire des fournitures que moy Antoine Despins Est faite a St Joseph. » (Diverses fournitures.) Signé Piquoté, La Jonquière, Bigot. f 30, p. 37.

118. Détroit, 29 juillet 1748. «Fournitures par antoine Cuillerier. 》 (Diverses fournitures.) Signé Longueuil, Navarre, Bigot. f 301, p. 36.

118. (Détroit), 30 juillet 1748. «Fournitures par Pierre Vallée. 》 (Diverses fournitures.) Signé Longueuil, Navarre, La Galissionière, Bigot. f 244, p. 318.

118. Michilimackinac, 30 juillet 1748. « Certificat. 》 (Fournitures.) Signé Le Gardeur de St. Pierre, La Galissionière, Bigot. f 293, p. 27. 118. Michilimackinac, 2 août 1748. «Memoire des fournitures que j'ay faites pour Le Roy par Lordre de monsieur de St. Pierre Commandant pour le Roy en ce Poste. » (Diverses fournitures.) Signé Duchesne, Le Gardeur de St. Pierre, La Galissionière, Bigot. f 276, p. 9.

118. Miamis, 3 août, 1748. «Memoire des fournitures faites par moy Louis de l'Isle au poste Des ouyatanons pour le Compte Et Service du Roy par L'ordre de Monsieur le Chevalier de La perade Commandant pour le Roy aud poste et suivant Les recommandations De monsieur Le Chevallier de Menthet Commandant au poste des Islinois qu'il Estoit ne cessaire de menager Les nations 8abache. 》 (Diverses fournitures.) Signé Louis de l'Isle, Lapérade fils, La Galissionière, Bigot. f 205-211, pp. 267-278.

118. Michilimackinac, 3 août 1748. «Memoire des fournitures que Jay fait par ordre de monsieur de St Pierre Commandant pour le Roy en ce Poste.» (Diverses fournitures.) Signé Duchesne, Le Gardeur de St. Pierre, La Galissionière, Bigot. f 275, p. 8.

118. Michilimackinac, 4 août 1748. «Etat des fournitures que La Toye a faite au Roy par ordres de Monsieur de St. Pierre Com. 
mandant en ce Poste. » (Diverses fournitures.) Signé Lajoie, La Gardeur de St. Pierre, La Galissionière, Bigot. f 298, p. 32.

118. Michilimackinac, 5 août 1748. «Certificat. » (Gages.) Signé Le Gardeur de St. Pierre, La Galissionière, Bigot. f 248, p. 323. 119. St. Joseph, 11 août 1748. « Certificat. » (Fournitures.) Signé Carqueville, La Jonquière, Bigot. f 26, p. 35 .

119. St. Joseph, 11 août 1748. « Certificat. 》(Fournitures.) Signé Carqueville, La Jonquière, Bigot. f 27, p. 35.

118. Détroit, 16 août 1748. «Certificat.» (Gages.) Signé Lon gueuil, La Galissionière, Bigot. f 277, p. 10.

118. Détroit, 16 août 1748. «Certificat.» (Gages.) Signé Lon gueuil, La Galissionière, Bigot. f 278, p. 111.

118. Détroit, 8 septembre 1748. «Fournitures de médicaments.» (Divers médicaments.) Signé Chapoton, Longueuil, La Galissionière, Bigot. f 347, p. 112.

118. Miamis, 30 septembre 1748. «Certificat. 》 (Fournitures.) Signé Lanaudière, La Galissionière, Bigot. f 411, p. 224.

118 Québec, 13 octobre 1748. «La Galissionière au Ministre. 》 (Sur les dépenses du Canada. Le Ministre peut être surpris qu'il demande qu'elles soient augmentées. Les propositions ont pour seul but l'agrandissement de la Colonie.) Signé La Galissionière. f 157. 158, pp. 208-209.

118. Miamis, 17 octobre 1748. «Certificat.» (Gages.) Signé Lanaudière, La Galissionière, Bigot. f 418, p. 230.

118. Miamis, 17 octobre 1748. « Certificat.» (Gages.) Signé Lanaudière, La Jonquière, Bigot. f 419, p. 231.

118. Miamis, 18 octobre 1748. «Certificat. 》 (Gages.) Signé Lanaudière, La Jonquière, Bigot. f 424, p. 236.

118. Québec, 20 octobre 1748. «Etat de la Depense que jay faite pendant les années $1746-1747$ et la presente année 1748 chez les sauvages des Cinq nations par ordre de Monsieur le Gouverneur Général pour le Service ainsy qu'il Ensuit. » (Divers services et 
fournitures.) Signé Loncaire, La Galissionière, Bigot. f 18, pp. 27-28.

118. Québec, 23 octobre 1748. «La Galissionière au Ministre.» (Sur le produit des postes et congés pour la présente année. Liste des gratifications accordées. Des réductions de gratifications peuvent être demandées. Les gratifications devraient être accordées indé. pendamment du produit. Une réforme dans l'allocation des gratifications est nécessaire. Les dépenses sont plus ou moins élevées en relation avec la location du poste.) Signé La Galissionière. f 148 . 151, pp. 191-194.

118. (Québec), 1748. «Etat des sommes provenantes du Produit des Postes du Pais d'En haut reçues par Monsieur de La Galissio niere tant de $\mathrm{M}$ de Beauharnois Suivant l'Etat de lui arresté le 14 octobre 1747, que des fermiers des Postes ou de Ceux aqui il a Eté Expédié des Congez pour y monter depuis L'arresté dud. Etat jusqu'a ce jour, Et des paie,ens faits sur les d sommes par le Tresorier de la Marine sur les ordres de Monsieur de La Galissioniere, ou manuellement par Mon d Sieur. » (Recettes : remis par Beauharnois des divers postes. Dépenses: aux divers commandants ou par gratifications.) f 152-158, pp. 195-207.

116-1. Québec, 25 octobre 1748. «Extrait du montant des Nouxeaux Droits perçûs au Bureau du Domaine d'occident en Canada pendant l'année et Jusqu'au cing cinq du mois d'octobre 1748 sur les Vins Eaux de vie et Guildives en Execution de l'arrest du Conseil d'Etat du 13 Janvier 1747. » (Sur le vin, l'eau-de-vie, guildive; à déduire les vins venus au compte du Roy; recette effective.) $\mathrm{Si}$ gné Cugnet, Nouchet, Lanoullier, Rivet, Dusouchet. f 76, pp. 120-121.

118. Miamis, 25 octobre 1748. « Certificat. 》 (Gages.) Lanaudière, La Jonquière, Bigot. f 438, p. 254.

119. Ouyatanons, 27 octobre 1748. «Certificat. » (Gages.) Signé Carqueville, La Galissionière, Bigot. f 66, p. 66.

119. Ouyatanons, 27 octobre 1748. «Certificat.» (Divers travaux.) Signé Carqueville, La Jonquière, Bigot. f 69, p. 69. 
119. Ouyatanons, 27 octobre 1748. «Certificat.» (Gages.) Signé Carqueville, La Jonquière, Bigot. f 74, p. 71.

119. Ouyatanons, 29 octobre 1748. «Certificat. 》 (Diverses fournitures.) Signé Carqueville, La Jonquière, Bigot. f 73, p. 70.

117-1. Québec, 31 octobre 1748. «Dépenses a Loccasion des Mouvemens des Pays d'En haut Exercice 1748. 》 (Fonds a remettre pour diverses fournitures.) Signé Varin, Hocquart. f 144146, pp. 261-267.

116-1. Québec, 1 novembre 1748. « Memoire au sujet de la situation des finances du Canada de l'Exercice de l'année mil sept cens quarante huit. » (Fonds ordonnés par l'état du Roy. Construction et radoub des bateaux et canots; courses et voyages; fret et voiture ; journées d'ouvriers; achats de marchandises; présents à faire aux Sauvages ; subsistance et remèdes; dépenses imprévues ; appointements des officiers généraux et autres; appointements des offlciers reformés; solde des compagnies; gages et entretien des employés dans les magasins et bureaux; loyers de magasins et bureaux; gratifications ordinaires, extraordinaires; préparatifs de guerre portés sur 1746 et 1747 ; fortifications, à déduire les dépenses portées sur 1745,1746 et 1747 ; autres diverses dépenses : portées sur 1746 ; total des fonds ordonnés. Dépenses données à Québec jusqu'au 30 septembre 1748 : construction et radoub de bateaux et canots; courses et voyages; fret et voiture ; journées d'ouvriers; achats de marchandises; achats de bois a brûler; hô pitaux ; dépenses imprévues ; appointements des officiers généraux et autres; appointements des officiers reformés; gages et entretien des employés; loyers de magasins et bureaux ; gratifications ordinaires, extraordinaires; dépenses pour les chantiers de construction : 1746, 1747 ; diverses dépenses pour la guerre; diverses dépenses pour l'Acadie; total de la dépense.) Signé Bigot. f 71.73, pp. $112-116$.

116-1. Québec, 1 novembre 1748. «Extrait des fonds necessaires pour le Payement de La Dépense faite a Quebec a L'occasion du nouveau chantier de construction des vaisseaux du Roy pendant Les Années 1746, 1747 et 1748. » (Dépenses suivant l'état en' 
voyé ; fonds ordonnés par l'état du Roy ; reste a ordonner.) Signé Bigot. f 77, p. 122.

116-1. Québec, 1 novembre 1748. «Estat de la Dépense qui a été faite a Quebec a L'occasion du Nouveau Chantier de Construction des Vaisseaux du Roy pendant Les années 1746, 1747 et 1748 ainsy qu'il En suit. » (Achats de bois; ouvrages de forgeron; aux charpentiers ; aux piqueurs ; diverses dépenses; achats de maisons et emplacements ; fonds accordés par l'état du Roy.) Signé Bigot. f 78, pp. 123-124.

116-1. Québec, 1 novembre 1748. «Extrait des fonds a ordonner par Suplement pour le parfait payement des dépenses en Canada a L'occasion de la guerre pendant Les années 1744 et 1746 audela de ceux ordonnés par les Etats de fonds du Roy pour les d. Exer cices. » (Pour le paiement des dépenses de 1744 et 1746.) Signé Bigot. f 81, p. 127.

116-1. Québec, 1 novembre 1748. «Bordereau des Depenses Genéralles dont les acquits ont été Payés par Le Trésorier de Quebec sur les fonds de l'Exercice de Lannée MVIIc Quarante huit depuis Le premier Janvier Jusques au dernier Septembre de la $\mathrm{d}$. année. 》 (Construction et radoub des bateaux et canots; courses et voyages ; Québec ; fret et voiture : Québec ; journées d'ouvriers et façons d'ouvrages; achats de marchandises et munitions ; marchandises et munitions : Québec, Montréal ; vivres : Québec, Montréal ; bois de chauffage : Québec, Montréal ; hôpitaux : Québec ; dépenses imprévues : Québec, Détroit, Michilimackinac ; détachement d'observation : Soulanges; Chateauguay, Isle Perrault, Lac des Deux Montagnes, Sault St. Louis, Senneville, La Prairie de la Madeleine, Chambly, Ste Thérèse, St. Jean, Chemin St. Jean; parties de guerre; appointements des officiers généraux et autres; appointements des officiers reformés ; solde de 28 compagnies; gages et entretien des employés dans les magasins et bureaux; loyers de magasins et bureaux ; gratifications ordinaires, extraordinaires; autres diverses dépenses; fortifications et réparations : bâtiments civils ; fortifications : Québec, rétablissement des casernes; réparations : Québec, Frontenac; construction du nouveau chantier; dépenses de guerre: Québec: achats de prisonniers, supplément de vivres 
aux prisonniers anglais et garde pour les prisonniers; traverses des rivières et dépenses pour les chevaux; diverses dépenses pour l'Acadie; dépenses de l'Isle Royale; récapitulation.) Signé Bigot. f $91-140$, pp. 144254 .

116-1. Québec, 2 novembre 1748. «Estat de la depense qui a été faite pour Réparations aux Battimens civils à Quebec et au fort frontenac pendant les neuf premiers Mois 1748. 》 (Québec: à divers charpentiers, menuisiers, maçons, serruriers, forgerons et ouvriers; Frontenac : à divers pour réparations.) Signé Bigot. f 79, p. 125 .

116-1. Québec, 2 novembre 1748. «Extrait des fonds necessaires pour Le payement des dépenses faites pour Les Réparations des Batimens Civils a Quebec et de fort frontenac pendant Les neuf premiers mois 1748. 》 (Dépenses suivant l'état joint à là lettre.) Signé Bigot, f 80, p. 126.

118. Québec, 2 novembre 1748. «Depenses des Pays d'en Haut 1748. 》 (Extrait du fonds à faire à Québec pour le paiement des dépenses de 1748.) Signé Bigot. f 177, p. 228.

116-1. Québec, 3 novembre 1748. «Extrait des fonds nécessaires pour le payement des Dépenses faites pour les fortiffications de la Ville de Quebec pendant Les neuf premiers mois mil sept cent quarante huit. 》 (Dépenses suivant l'état; à déduire les revenus provenant de l'augmentation des droits; reste à remettre.) Signé Bigot. f 74, p. 117.

116-1. Québec, 3 novembre 1748. «Estat de la Dépenses qui a été faite a Quebec aux fortifications de la d't Ville pendant Les neuf premiers mois 1748. » (Fortifications : rétablissement des casernes; total.) Signé Bigot. f 75, pp. 118-119.

116-1. Québec, 3 novembre 1748. «Extrait des Fonds a ordon ner pour le parfait payement des dépenses faites en Canada a L'oc' casion de la guerre pendant Les Années 1743, 1745 Et 1747 au dela de ceux ordonnés par Les Etats de fonds du Roy pour Lesd. Exercices. 》 (Pour le payement des dépenses des années 1743, 1745 et 1747.) Signé Bigot. f 141, p. 255. 
116-1. Québec, 4 novembre 1748. «Dépenses a Loccasion de la Guerre. » (Fonds nécessaires au delà de ceux ordonnés.) Signé Bigot. f 70, pp. 110-111.

118. Québec, 4 novembre 1748. «Bigot au Ministre.» (Sur les dépenses des pays d'en haut. Il y avait de grands abus dans les fournitures. Il y a eu, dans le passé, de la collusion entre les mar. chands et les commandants des postes. La Galissionière en a connaissance, mais celui-ci demande que le système ne change pas car les officiers sont pauvres. Envoie l'extrait des fonds a remettre pour 1749.) Signé Bigot. f 258-259, pp. 346-347.

118. Miamis, 17 novembre 1748. «Certificat.» (Fournitures.) Signé Lanaudière, La Galissionière, Bigot. f 410, p. 223.

119. Ouyatanons, 21 novembre 1748. «Certificat. 》 (Gages.) Signé Carqueville, La Jonquière, Bigot. f 65, p. 65.

119. Ouyatanons, 21 novembre 1748. «Certificat. 》 (Fournitures.) Signé Carqueville, La Galissionière, Bigot. f 68, p. 68 .

119. Ouyatanons, 21 novembre 1748. «Certificat. 》 (Gages.) Si, gné Carqueville, La Jonquière, Bigot. f 75, p. 71.

119. Ouyatanons, 24 novembre 1748. «Certificat. 》 (Estimation et achat de maison.) Signé Henri Catin, Drouet de Carqueville, Bigot. f 70, p. 69.

119. Ouyatanons, 25 novembre 1748. «Certificat. 》 (Gages.) Signé Drouet de Carqueville, La Galissionière, Bigot. f 67, p. 67.

119. Ouyatanons, 27 novembre 1748. «Certificat. 》 (Fournitures.) Signé Lanaudière, La Jonquière, Bigot. f 72, p. 70.

119. Michilimackinac, 26 juillet 1749. «Memoire des Marchandises que Jay fourny moy Jasmin pour le Roy par ordre de $\mathrm{Mr}$ de St Pierre Commend' Pour le Roy a Missilimakinac livré En mil spet cent quarante huit. » (Diverses fournitures.) Signé Baptiste Jasmin, Le Gardeur de St. Pierre, La Jonquière, Bigot. f 182-183, pp. $172-174$.

116-1. Québec, 24 octobre 1750. «Etat des fonds faits par les Etats du Roy du Montant des Recettes Extraordinaires et des dépenses 
faites en Canada sue les Exercices de 1746, 47, 48 et 1749 pour servir à Constater les Excedents de depenses de chacun de ces Exercices. 》 (Fonds faits par les états du Roy des années 1748, 1749 et 1750 . Récapitulation. Total de la recette. Dépenses. Sommaire.) Signé Bigot. f 238-245, pp. 409-424.

116-2. Québec, 26 octobre 1750. «Extrait du fonds a ordonner par suplement pour le parfait payement des dépenses génerales faites en Canada pendant L'année 1748. 》 (Dépenses; à déduire les fonds ordonnés sur l'exercice de 1748, 1749, 1750 ; fonds à faire.) Signé Bigot. f 334, p. 207.

Voir aussi : 1745. 115-2. Québec, 19 septembre 1748. "Bordereau... $1745 \ldots$ f 227 , p. 162 .

Voir aussi : 1747. 117-1. Québec, 18 septembre 1747. «Reparztions... 1748... \& 156 , p. 285.

Voir aussi : 1747. 117-1. Québec, 15 octobre 1747. «Fortification 1747. 》f 163, p. 299.

Voir aussi : 1747. 117-1. Québec, 31 octobre 1747. «Dépenses... $1748 \ldots \gg$ f $321-323$, pp. $175-180$.

Voir aussi : 1747. 117-1. Détroit, 17 juillet 1748. «Memoire... Chauvin... 》f 123-126, pp. 235-242.

Voir aussi : 1747. 117-1. Québec, 22 octobre 1748. «Dépenses... $1748 \ldots \gg$ f 155 , pp. $283-284$.

Voir aussi : 1747. 116-1. 23 octobre 1748. «Etat des sommes... 》 f 144150 , pp. $258-268$.

Voir aussi : 1745. 115-2. Québec, 30 octobre 1748. « Memoire... $1745 \ldots \gg$ f 251 , p. 212.

Voir aussi : 1746. 116-1. Québec, 31 octobre 1748. « Memoire... MVIIc Quarante six... »f 89-90, pp. 141-143.

Voir aussi : 1749. 118. Québec, 31 octobre 1748. « Canada 1748... Exercice 1749. 》f 178-185, pp. 229-244.

Voir aussi : 1747. 115-2. Québec, 3 novembre 1748. « Memoire... » f $365-366$, pp. $413 ; 414$. 
Voir aussi : 1749. 119. Ouyatanons, 1 mai 1749. « Memoire... 》 f $80-81$, pp. 76-78.

Voir aussi : 1749, 116-1. Versailles, 10 mai 1749. «... Canada... Dom'e... »f 211, p. 367.

Voir aussi : 1749. 119. Ouyatanons, 14 mai 1749. « Memoire... » f 79, p. 75.

Voir aussi : 1749. 119. Michilimackinac, 16 mai 1749. «Etat... » f 62, p. 63 .

Voir aussi : 1749. 119. Ouyatanons, 16 mai 1749. «Memoire... 》 f 77 , pp. $73 \cdot 74$.

Voir aussi : 1749. 118. Miamis, 1 juin 1749. « Memoire... » $f$ 425. 427, pp. $237-240$.

Voir aussi : 1749. 118. Détroit, 2 juin 1749. « Memoire... »f 371 , pp. 136-137.

Voir aussi : 1749. 118. Détroit, 6 juin 1749. « Memoire... »f 370 , p. 135 .

Voir aussi : 1749. 118. Miamis, 7 juin 1749. «Fournitures... »f 429.431, pp. 243-246.

Voir aussi : 1749. 119. A la Baye, 14 juin 1749. «Fournitures... » f $12 \cdot 14$, pp. $15-19$.

Voir aussi : 1749. 119. A la Baye, 14 juin 1749. «Fournitures... » f $15-19$, pp. $20-26$.

Voir aussi : 1749. 119. Détroit, 28 juin 1749. «Fournitures... 》 f 230 , p. 221.

Voir aussi : 1749. 118. Détroit, 30 juin 1749. «Etat... »f 377 , p. 144.

Voir aussi : 1749. 119. St. Joseph, 4 juillet 1749. « Memoire... » f 31, p. 38.

Voir aussi : 1749. 118. Détroit, 16 juillet 1749. «Memoire...» f 399, pp. 211-212.

Voir aussi : 1749. 118. Détroit, 18 juillet 1749. «Compte...» f 401, p. 214. 
Voir aussi : 1749. 118. Détroit, 21 juillet 1749. «Fournitures... 》 f 405 , p. 210 .

Voir aussi : 1749. 118. Détroit, 25 juillet 1749. «Memoire... » f $380-381$, pp. $149 \cdot 152$.

Voir aussi : 1749. 118. Détroit, 25 juillet 1749. « Memoire... » $f$ 385-390, pp. $189-202$.

Voir aussi : 1749. 119. Détroit, 26 juillet 1749. « Fournitures... » f 228 , p. 218.

Voir aussi : 1749. 116-1. (Québec), 17 septembre 1749. «Etat des sommes... 1748... 1749... » f 151-161, pp. 269-285.

Voir aussi : 1749. 116-1. Québec, 24 octobre 1750. « Bordereau... Tachereau... 1749... »f 272, p. 37.

Voir aussi : 1749. 116-2. Québec, 24 octobre 1750. «Balance des fonds... » f 332-335, pp. 201-205.

Voir aussi : 1746. 116-2. Québec, 26 octobre 1750. «Extrait... 1746. 》f 336, p. 209.

Voir aussi : 1746. 115-2. Québec, 24 octobre 1751. «Bordereau... 1746. 》f $327-328$, p. 342.

\author{
Cameron NISH, \\ professeur à l'Université Sir George Williams \\ et \\ directeur de recherche, \\ Centre de Recherche en Histoire économique \\ du Canada français.
}

